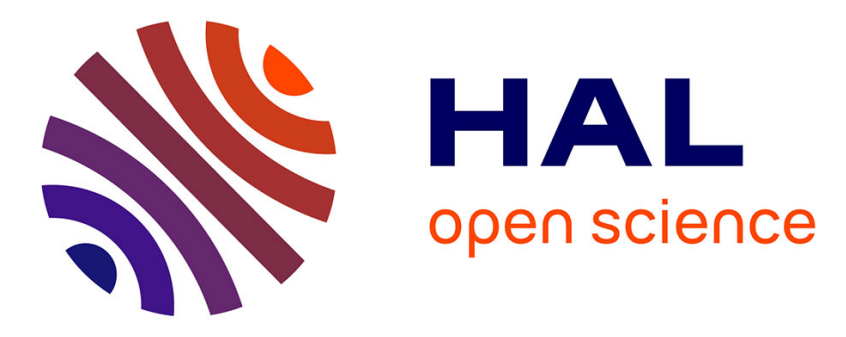

\title{
Atypical reversed pressure-induced phase transformation in Ge nanowires
}

\author{
Doriane Djomani, Francesco Capitani, Jean Blaise Brubach, Eugenio \\ Calandrini, Charles Renard, Daniel Bouchier, Jean Paul Itié, Pascale Roy, \\ Laetitia Vincent
}

\section{To cite this version:}

Doriane Djomani, Francesco Capitani, Jean Blaise Brubach, Eugenio Calandrini, Charles Renard, et al. Atypical reversed pressure-induced phase transformation in Ge nanowires. Nanotechnology, 2020, 31 (23), pp.235711. 10.1088/1361-6528/ab7b06 . hal-03004890

\section{HAL Id: hal-03004890 https://hal.science/hal-03004890}

Submitted on 17 Nov 2020

HAL is a multi-disciplinary open access archive for the deposit and dissemination of scientific research documents, whether they are published or not. The documents may come from teaching and research institutions in France or abroad, or from public or private research centers.
L'archive ouverte pluridisciplinaire HAL, est destinée au dépôt et à la diffusion de documents scientifiques de niveau recherche, publiés ou non, émanant des établissements d'enseignement et de recherche français ou étrangers, des laboratoires publics ou privés. 


\section{Atypical reversed pressure-induced phase transformation in Ge nanowires}

Doriane Djomani*, Francesco Capitani $\uparrow$, Jean Blaise Brubach $\uparrow$, Eugenio Calandrini $\uparrow$, Charles Renard*, Daniel Bouchier*, Jean Paul Itié $\dagger$, Pascale Roy†, Laetitia Vincent*\&

* Centre de Nanosciences et Nanotechnologies (C2N), CNRS, Univ. Paris-Sud, Université Paris-Saclay, 10 Boulevard Thomas Gobert, 91120 Palaiseau France

$\dagger$ Synchrotron SOLEIL, L’orme des Merisiers, 91190 Gif-sur-Yvette Cedex, France

\& corresponding author: laetitia.vincent@u-psud.fr

KEYWORDS. Phase transformation; pressure diamond anvil cell; nanowires; Raman spectroscopy; infrared absorption 


\section{ABSTRACT:}

Phase transformations of Ge under compression/decompression cycle at room temperature were studied in a diamond anvil cell (DAC) using in-situ synchrotron X-ray diffraction, Raman spectroscopy and near infrared absorption techniques. Upon compression similar behavior is observed in nanowires and bulk although a higher stability is observed in nanowires. The cubic-diamond phase $(\mathrm{Ge}-3 \mathrm{C})$, the most energetically favorable phase, transforms into the $\beta$-tin metallic phase at high pressure and the reverse Ge- $\beta$-tin to Ge-3C transformation is generally inhibited by kinetics when pressure is released. While the transformation in Ge bulk leads mostly to Ge-ST12 phase, the loading/unloading cycle of Ge nanowires in DAC leads back to Ge-3C, exhibiting unprecedented size effects. A comprehensive characterization of the final states is described.

\section{INTRODUCTION}

There has been a renewed interest in the last few years for the synthesis of Si and Ge allotrope structures offering modified band gap or innovative heterostructures for band engineering in group IV semiconductors. In $\mathrm{Si}$ and $\mathrm{Ge}$ nanowires, we have developed a process to produce hexagonal $2 \mathrm{H}$ phases along the $3 \mathrm{C}$ nanowires $[1,2]$. The unveiled martensitic transformation occurring under shear stress suggests new mechanisms of deformation-induced phase transformation at the nanoscale.

In recent years, theoretical and experimental studies showed that mechanical properties are significantly modified at the nanoscale due to surface effects. Nanowires generally exhibit modified mechanical properties with decreasing diameter size, especially in the elastic regime - i.e. higher stiffness, larger yield stress, lower compressibility- [3, 4]. For instance bulk modulus was reported to increase by $25 \%$ in Si nanowires (diameter of $70 \mathrm{~nm}$ ) [5]. Atomistic simulations have shown that intrinsic surface stresses are responsible for such phenomena because they induce substantial intrinsic compressive stresses that may lead to spontaneous reorientations or phase transformations. Additionally, edges on free surfaces form high stress 
points and they can act as preferential nucleation site for defects and/or shear failure. Deformation mechanisms (dislocation formation, slip and twinning) can thus differ significantly from the bulk and can cause unexpected phase transformation pathway in nanowires.

Bulk crystalline germanium is known to exhibit several allotropic phases under compression and decompression [6,7]. Most of the allotrope phases obtained by static compression of a sample in a high-pressure diamond anvil cell (DAC) have been identified by means of in-situ measurements of electrical resistivity, X-ray diffraction and Raman spectroscopy in the last forty years. The cubic diamond phase 3C (Ge-I) transforms into the metallic $\beta$-tin phase (Ge-II) under hydrostatic compression conditions around $10 \mathrm{GPa}[8,9]$. The pressure range where this transformation takes place is larger when hydrostatic conditions are not fulfilled and shear stress is involved: the transformation threshold is lowered to $7 \mathrm{GPa}$ if compression is performed without any pressure transmitting medium [10] and is completed around $12 \mathrm{GPa}[9,10]$. The $\beta$-tin phase of Ge is then stable up to $75 \mathrm{GPa}$. The decompression leads to the formation of distinct metastable phases denser than the $3 \mathrm{C}$ form, even though they display a higher energy [11]. Kinetics of transformation (i.e. enthalpy barrier) may account for this irreversibility (see in particular figure $2 \mathrm{f}$ in ref [12] showing that enthalpy barrier of Ge- $\beta$ Tin $\rightarrow \mathrm{Ge}-3 \mathrm{C}$ is larger).

The decompression leads mainly to a metastable tetragonal phase ST12 (referred as Ge-III). The same phase was identified upon pressurization and decompression of amorphous Ge (a-Ge) [13]. However, depending on the unloading conditions (hydrostaticity, kinetics, temperature) another metastable cubic phase BC8 (Ge-IV) can be obtained. At room temperature, Ge-BC8 is very unstable and transforms into the hexagonal $2 \mathrm{H}$ structure $(\mathrm{Ge}-\mathrm{V})$. The phases formed after decompression, Ge-ST12 and Ge-BC8, are still under debate. In fact, both transformations Ge- $\beta$ tin $\rightarrow$ Ge-ST12 and Ge- $\beta$ tin $\rightarrow$ Ge-BC8 exhibit similar energetic barriers [12], and both structures are energetically equivalent [11, 12, 14]. Note that another 
intermediate phase Ge-R8 was observed solely by Haberl et al. [15] and no calculations are available regarding the barrier of Ge- $\beta$ tin $\rightarrow$ Ge-R8.

Experimentally, transition pathways depend strongly on the unloading conditions. In most cases, the ST12 phase is formed. However, BC8 is expected for very fast unloading [16] and low temperature [17, 18]. According to Haberl et al. [15] the critical parameter dominating the transition pathway is the local stress environment. They showed that BC8 can be formed if very strict hydrostaticity is fulfilled. However, the total absence of shear is quite difficult to obtain in DAC. It is important to note that the control of the decompression pathway is challenging and no consensus appears in the literature still after 30 years of studies.

The size-dependency of the phase transformation is another important parameter to investigate. The purpose of these experiments was to clarify the different transformation pathways of Ge- $\beta$-tin phase during decompression and evidence how nanoscale effects may affect the transformation in Ge nanowires. Therefore, size effects were examined by comparing the decompression pathways in Ge bulk and in nanowires (40 $\mathrm{nm}$ in diameter).

\section{EXPERIMENTAL SECTION}

Sample synthesis

N-type Ge with a resistivity on the range 1-9 $\Omega . \mathrm{cm}$ was employed for bulk measurement. For optical measurements, round pellets with a diameter of $250 \mu \mathrm{m}$ and a thickness of around $20 \mu \mathrm{m}$ was shaped and mechanically polished from a $<111>$-oriented Ge substrate. For XRD measurements, a powder of microcrystallites was obtained by milling in ethanol a piece of the aforementioned substrate.

Ge nanowires were synthesized by VLS growth process using ultra-high vacuum chemical vapor deposition. Details of the experimental procedure were described previously [19]. We have used nanowires of about $2 \mu \mathrm{m}$ length and a mean diameter of $40 \mathrm{~nm}$. After the 
growth, gold catalysts were removed by chemical etching with $\mathrm{KI}: \mathrm{I}_{2}: \mathrm{H}_{2} \mathrm{O}$ solution. Ge nanowires have been scrapped off from their growth substrate with a scalpel blade and inserted in the DAC with a pressure transmission medium. The nanowires with the pressure medium $(\mathrm{KBr}, \mathrm{NaCl}$ or $\mathrm{PE})$ form a heap that will be compressed to a final sintered pellet.

\section{High pressure set-up}

Two membrane diamond anvil cells (DAC) with a culet surface of $500 \mu \mathrm{m}$ diameter were used as pressure device ( $300 \mu \mathrm{m}$ for XRD measurements). The samples were loaded into $150 \mu \mathrm{m}$ hole drilled in a preindented stainless steel gasket with a thickness of $50 \mu \mathrm{m}$. Different pressure transmitting media were employed depending on the performed characterizations and the transparency of the media to the wavelength range. Polyethylene (PE) and Ne were used for XRD measurements to check the effect of hydrostaticity. PE or $\mathrm{NaCl}$ were used for Raman measurements and $\mathrm{KBr}$ was used for infrared absorption measurements. For pressure monitoring, a ruby crystal or gold powder (in the case of XRD) were inserted in the DAC. The highest pressure attained was about $20 \mathrm{GPa}$. Compression/decompression cycles were carried out at room temperature. Decompression rate was about $1 \mathrm{GPa} / \mathrm{min}$ either for bulk and nanowire measurements. After decompression the pellets can be handled for further analyses. Samples were for instance prepared by focused ion beam for transmission electron microscopy (see supporting information SI).

\section{Diffraction set-up}

Psiché beamline at synchrotron SOLEIL is partially dedicated to diffraction under extreme conditions. The X-ray diffraction pattern is recorded using a CCD detector (Marccd) or a flat panel (Perkin Elmer) detector. The high-pressure cell is mounted on a goniometer which allows to rotate the cell during acquisition. A ruby luminescence set-up is installed on the beamline in order to measure pressure. The pressure is increased using a membrane connected to a helium bottle under pressure. The x-rays are collimated down to $80 \mu \mathrm{m}$ using 
two pairs of slits. The monochromatic beam is obtained using a double crystal monochromator with Si-(111) crystals and the beam is focused vertically with a large vertical mirror down to $100 \mu \mathrm{m}$. To obtain powder diffraction data the sample used in that case was beforehand milled as described previously.

\section{Raman set-up}

Raman spectra were collected using a Horiba iHR320 spectrometer equipped with a 1800 grooves $/ \mathrm{mm}$ grating, a Peltier cooled CCD, a red solid state laser $(632 \mathrm{~nm})$ as excitation source, a fiber coupled Horiba Raman Head with a 50x objective and an edge filter to reject the Rayleigh line in the backscattering geometry. Raman spectra were calibrated using the emission lines of a Ne lamp.

\section{Optical set-up}

Infrared absorption spectra were recorded by means of a Bruker 125 spectrometer coupled to a custom high pressure setup [20]. The setup features a couple x15 Cassegraintype objectives to focus the radiation onto the sample and a system of motorized translations and flip mirrors allow the continuous control of the alignment under vacuum. The pressure applied on the sample was determined by in situ measuring the ruby fluorescence. To match the spectral range of Ge gap, the experimental setup exploited the tungsten internal source, a Quartz beamsplitter and the InSb detector. An aperture of $5 \mathrm{~mm}$ was focused by the Cassegrain to create a light spot onto the 250 microns area of the sample inside the hole of the gasket. Absorption spectra were determined by the following relation $A=-\log \left(I_{s} / I_{0}\right)$, where $I_{s}$ and $\mathrm{I}_{0}$ are the light intensities transmitted by the sample and the empty DAC, respectively.

\section{RESULTS and DISCUSSION}

Structure and pathway determined by XRD 
In situ compression/decompression XRD measurements have been performed to monitor the evolution of the lattice parameters as a function of the pressure and to reveal the formation of new phases through the appearance and disappearance of Bragg peaks (figure 1). In bulk, when increasing the pressure, the expected Ge-3C to $\beta$-tin phase transformation occurred around $8.5 \mathrm{GPa}$ as indicated by the emergence of the (200) diffraction peak of Ge- $\beta$ tin. The transition onset was the same for $\mathrm{Ne}$ and PE transmitting media indicating similar (non)-hydrostatic conditions. In the 8.5-12 GPa pressure range, $3 \mathrm{C}$ and $\beta$-tin phases coexisted (figure 1). Increasing pressure up to $15 \mathrm{GPa}$ led to the total disappearance of the main (111) peak of $\mathrm{Ge}-3 \mathrm{C}$ and thus confirmed the purity of the $\mathrm{Ge} \beta$-tin phase. The pressure was then stabilized over 12 hours.

Shortly after the decompression that occurred in a couple of seconds (ultra-fast decompression only in that case), we observed the presence of a mixture of Ge-ST12 and a small quantity of Ge-BC8 as shown in figure 2. Similar spectrum was obtained 24 hours later, although the amount of Ge-BC8 seemed to decrease with time according to the reduction of the intensity of the peak corresponding to the Bragg reflection on the (211) planes of Ge-BC8 at $7.57^{\circ}$. This suggested the transformation to the expected $2 \mathrm{H}$ phase, nonetheless we did not identify any $2 \mathrm{H}$ diffraction peak in the spectrum probably due to the superimposition of the expected low intensity $2 \mathrm{H}$ peaks with the Ge-ST12 peaks and from the gasket. For lower rate decompression $(1 \mathrm{GPa} / \mathrm{min})$, performed with PE transmitted medium, only Ge-ST12 was found. It is worth noticing, the absence of $\mathrm{Ge}-3 \mathrm{C}$ on all the spectra after the decompression.

Ge nanowires inserted in the DAC with Ne were loaded up to $17.5 \mathrm{GPa}$. From the diffraction patterns (Figure 3) the lattice parameter and the unit-cell volume of Ge-3C in nanowires were obtained. In supporting information, figure SI.1 illustrates the evolution of the crystal cell volume as a function of pressure enabling to obtain respectively the isothermal bulk modulus $\mathrm{B}_{0}$ and its first pressure derivative $\mathrm{B}^{\prime}{ }_{0}$. We found $\mathrm{B}^{\prime}{ }_{0}=4$ and $\mathrm{B}_{0}=73.8 \mathrm{GPa}$ 
which are in well agreement with the experimental values reported for bulk [9, 21, 22]. Thus, contrarily to results obtained from Ge nanocrystallines [23], no size effect is observed here on the compressibility of the Ge nanowires down to a diameter of $40 \mathrm{~nm}$.

Above $12 \mathrm{GPa}$, the phase transformation is evidenced in figure 3 by the emergence of the (200) and (101) Bragg diffraction peaks of Ge- $\beta$-tin phase and the concomitant intensity reduction of the Ge-3C peaks (see the indexed peaks). A coexistence of Ge-3C and Ge- $\beta$-tin phases was reported up to $15 \mathrm{GPa}$. The transformation range for nanowires (12-15 GPa) is therefore shifted towards higher pressure compared to their bulk counterpart under the same loading conditions although bulk modulus derived from P-V measurements is comparable to the reference value of bulk. This result suggests a higher stability for nanometric size.

The pressure was maintained at $18 \mathrm{GPa}$ for 12 hours to ensure the total transformation and stabilization of the Ge- $\beta$-tin compressed structure. After unloading, the pattern (figure 3) evidenced the restoration of diffraction peaks that can be indexed to Ge-3C. Thus Ge- $\beta$-tin phase in nanowires go back to $3 \mathrm{C}$ structure after decompression as opposed to the bulk. This may be attributed to size effects on the transition kinetics.

\section{Raman pathway determination}

As a complementary characterization, we have used in-situ Raman spectroscopy to probe vibrational dynamics under pressure in order to confirm the differences in decompression pathways between bulk and nanowires. Samples were loaded in the DACs with $\mathrm{NaCl}$ pressure medium. Raman spectra were recorded up to $14 \mathrm{GPa}$ (figure $4 \mathrm{a}$ ). The first order Raman peak of the Ge-3C structure, corresponding to the $\mathrm{F}_{2 \mathrm{~g}}$ mode, was found at 304 $\mathrm{cm}^{-1}$ indicating a small pressure induced by the DAC setting. Indeed, this band shifted from $300 \mathrm{~cm}^{-1}$ value at $0 \mathrm{GPa}$ [24] to higher frequency with pressure. At $\mathrm{P}=10 \mathrm{GPa}$, the peak intensity increased and the band edge opens up to about $2 \mathrm{eV}$ [25], corresponding to the energy of the excitation laser $(632 \mathrm{~nm})$, thus giving rise to a resonant Raman effect. 
Compression led ultimately to the disappearance of the $\mathrm{F}_{2 \mathrm{~g}}$ band indicating that the $\mathrm{Ge}-3 \mathrm{C}$ has been totally transformed. Above $12 \mathrm{GPa}$, the spectra were dominated by a new broad band at low frequency $220 \mathrm{~cm}^{-1}$ corresponding to the TO phonon mode of the compressed Ge- $\beta$-tin [26]. This peak also presented a blue shift with increasing pressure.

During decompression, spectra compatible with the Ge- $\beta$-tin phase remained down to $8 \mathrm{GPa}$. There was clear evidence for the transformation toward Ge-ST12 with the appearance of the six characteristic peaks in the 150 to $350 \mathrm{~cm}^{-1}$ range $[27,28]$ as shown in figure $4 \mathrm{~b}$.

For comparison, figure 5 shows the first order Raman spectra on compressed and decompressed Ge nanowires. Under compression, the main $F_{2 g}$ peak was blue shifted with a progressive increase of FWHM from 10.5 to $12.5 \mathrm{~cm}^{-1}$. The TO peak of the $\beta$-tin structure appeared above $9.5 \mathrm{GPa}$ and a mixture of both Ge-3C and Ge- $\beta$-tin was observed up to 13 $\mathrm{GPa}$. The complete phase transition was unveiled by the disappearance of the $\mathrm{F}_{2 \mathrm{~g}}$ mode at 14 GPa. The transformation onset was thus similar for both nanowires and bulk but the coexistence pressure range of $\mathrm{Ge}-3 \mathrm{C}$ and $\mathrm{Ge}-\beta$-tin was larger for nanowires, although this range was reduced using $\mathrm{NaCl}$ instead of $\mathrm{Ne}$ (used for XRD measurements - suggesting that shear stresses reduce the pressure range of transformation). Following the decompression, the $\beta$-tin phase appeared stable down to $9 \mathrm{GPa}$. At $4.7 \mathrm{GPa}$, the $\mathrm{F}_{2 \mathrm{~g}}$ mode of the Ge-3C phase reappears at $321.4 \mathrm{~cm}^{-1}$. The broad band around $270 \mathrm{~cm}^{-1}$ is characteristic to an amorphouslike phase during decompression. After complete unloading, the $\mathrm{F}_{2 \mathrm{~g}}$ peak is finally located at $307 \mathrm{~cm}^{-1}$ indicating a compressive strain in the final state. From this decompressed bulk pellet, XTEM sample was prepared using focused ion beam milling. Ge-ST12 is clearly evidenced by the SAED pattern in the $<110>$ zone axis in figure SI.2 of supporting information. The sample remains monocrystalline with the appearance of some fractures.

Optical results in the near infrared 
Figure 6 shows the absorption coefficient spectra recorded in bulk before compression and after decompression unveiling clearly the irreversibility. There is no ambiguity on the difference of optical inter-band absorption between both structures Ge-3C and Ge-ST12 (Fig. 6a). For Ge-3C, the allowed direct transition obtained from Tauc plot analysis is measured at $0.9 \mathrm{eV}$ at an initial pressure of $0.03 \mathrm{GPa}$ in somewhat moderate agreement with the generally accepted value of $0.82 \mathrm{eV}[29,30]$. For Ge-ST12 after release, Tauc plot analyses illustrated in figure 6 (b and c) provide an indirect allowed and a direct allowed transitions with an energy of $0.53 \mathrm{eV}$ and $0.73 \mathrm{eV}$ respectively. These measurements agree with recently reported results of $0.59 \mathrm{eV}$ and $0.74 \mathrm{eV}$ from Zhao et al. [28]. Note that a pressure between 2 or $3 \mathrm{GPa}$ is generally retained in the DAC after a cycle of compression and decompression present for all the experiments carried out, such residual stress may therefore lead to an overestimation of the gap values in the final state.

Absorption coefficients measured in NWs before and after the compression/decompression cycle are shown in figure 7. Before compression, the direct energy transition was calculated by Tauc plot analysis at $0.8 \mathrm{eV}$. After decompression, Tauc analysis allows to estimate a direct gap value of $0.9 \mathrm{eV}$ which is in agreement with Ge-3C with some residual pressure [30] (note that a value of 2 or $3 \mathrm{GPa}$ was estimated with the ruby fluorescence after decompression). This confirms again the reversible transition in nanowires. The transformation cycle has formed a polycrystalline Ge-3C pellet (as shown in SI.3).

\section{Discussion}

Under compression in DAC with identical conditions, Ge bulk and bundles of Ge nanowires $(\phi=40 \mathrm{~nm})$ experience similar behavior although a higher stability is observed in NWs. This higher stability may be explained by a possible lower shear stress or size effect.

As expected, the loading/unloading transformation cycle at room temperature is irreversible in bulk. In most cases the decompression from the pressurized $\beta$-tin phase is the metastable Ge-ST12 phase. The studies reported in bulk showed the importance of decompression rate and shear stresses for the phase transformation during release. The shear 
strain in DAC generally enhances the formation of ST12-Ge as described in [15], and also in the high-pressure torsion experiments [31]. The high rate enables the formation of $\mathrm{BC} 8[15$, 16]. With our experimental conditions, BC8 was only observed after very fast decompression (1 GPa/s) within a mixture with Ge-ST12. No trace of 3C phase was detected in bulk whatever the decompression conditions. This irreversible behavior may be controlled by kinetics of the transformation pathways as demonstrated by Wang et al. [12].

In contrast, XRD, Raman and infrared spectroscopy clearly evidence a different pathway in Ge nanowires; the structure goes back to Ge-3C and no trace of Ge-ST12 was observed. For these measurements, same decompression rate $(1 \mathrm{GPa} / \mathrm{s})$ was used for bulk and nanowires. Indeed, in the case of a nanowire heap filling the DAC, shear stresses are also introduced as a consequence of bridging the diamond culets as well as direct interactions between nanowires. For the case of Ge bulk, decompression rate and shear stress had no effect on the "reversible" formation of Ge-3C. Then the major difference in phase transformation kinetics is ascribed here to size effects. As described in ref. [12] Ge-3C may be realized when the system has a chance to overcome the kinetic barrier. Our observations suggest that interface energies may increase the total energy of the system and in turn modifies kinetics of transition under decompression. To model this effect, further experiments are required to gain a comprehensive knowledge as a function of nanowire size.

\section{Conclusion}

We have evidenced a size dependence on the phase transformation pathways under decompression of Ge bulk and nanowires. The decompression of bulk leads to the formation of ST-12 structure while in nanowires the structure returns back to the initial standard cubic diamond phase 3C. Phases were fully identified by XRD, Raman and near-infrared spectroscopy. Moreover, NIR absorption provided the optical absorption edge in Ge-ST12 phase. The reversal transformation to Ge-3C in NWs may be accounted for an effect of 
interface energy on the transformation kinetic. Further experiments coupled with theoretical simulations could help in understanding the size effect on the phase transitions.

\section{Acknowledgement}

This work was supported by $\mathrm{PhD}$ grant and funding from the LIDEX Nanodesign plateform and in the framework of the ANR HEXSIGE project (ANR-17-CE030-0014-01) funded by the French Agence Nationale de la Recherche.

Samples were performed in micro/nano fabrication facility of $\mathrm{C} 2 \mathrm{~N}$, which is part of the national network Renatech of French clean rooms centers. 
Figure caption

Figure 1: XRD patterns of Ge bulk powder under compression at room temperature up to 15 $\mathrm{GPa}$ in $\mathrm{Ne}$ transmitting pressure medium. The X-ray wavelength was $0.3738 \AA$.

Figure 2: XRD patterns of Ge bulk powder after decompression at room temperature from Ge- $\beta$-tin structure. The X-ray wavelength was $0.3738 \AA$. After a fast decompression $(\mathrm{GPa} / \mathrm{s})$ Ge-ST12 and Ge-BC8 are present.

Asterisk indicates an unresolved peak that could be composed of two peaks one arising from the gasket.

Figure 3: XRD patterns of Ge nanowires under compression up to $17.5 \mathrm{GPa}$ and after decompression at room temperature in $\mathrm{Ne}$ transmitting pressure medium. The X-ray wavelength was $0.3344 \AA$. After unload, the pressure was around $0.5 \mathrm{GPa}$.

Asterisks in yellow, blue and grey identify peaks from resp. Au, Ne and stainless-steel gasket.

Figure 4: Raman spectra in the range $200-530 \mathrm{~cm}^{-1}$ recorded on bulk Ge for different values of pressure up to $14 \mathrm{GPa}$ in (a) and after decompression in (b). Ge-ST12 peaks are indexed in the upper part of $(b)$.

Figure 5: Raman spectra on Ge NWs (a) under compression and (b) after decompression.

Figure 6: (a) absorption coefficients extracted from transmittance measurements for Ge-3C bulk before compression and for Ge-ST12 after decompression (2 GPa). Derived Tauc's plot of Ge-ST12 (b) for indirect allowed optical transition (c) for direct allowed optical transition. The values of the gap energies are obtained from the linear fits and correspond to the energy value at zero absorption. (In a sake of clarity of the curve, the origin of the $y$-axis in plot $b$ is not zero, and the gap value has to be extrapolated the exact value is $0.53 \mathrm{eV}$ ).

Figure 7: (a) absorption coefficients extracted from transmittance measurements for Ge-3C NWs before compression and after decompression. 
[1] Vincent L, Djomani D, Fakfakh M, Renard C, Belier B, Bouchier D and Patriarche G 2018 Shear-driven phase transformation in silicon nanowires Nanotechnology 29 125601

[2] Vincent L, Patriarche G, Hallais G, Renard C, Gardès C, Troadec D and Bouchier D 2014 Novel Heterostructured Ge Nanowires Based on Polytype Transformation Nano Letters 14 4828-36

[3] Gerberich W W, Michler J, Mook W M, Ghisleni R, Östlund F, Stauffer D D and Ballarini R 2011 Scale effects for strength, ductility, and toughness in "brittle" materials Journal of Materials Research 24 898-906

[4] Ngo L T, Almecija D, Sader J E, Daly B, Petkov N, Holmes J D, Erts D and Boland J J 2006 Ultimate-strength germanium nanowires Nano Lett 6 2964-8

[5] Wang Y, Zhang J, Wu J, Coffer J L, Lin Z, Sinogeikin S V, Yang W and Zhao Y 2008 Phase Transition and Compressibility in Silicon Nanowires Nano Letters 8 2891-5

[6] Gogotski Y and Domnich V 2003 High Pressure Surface Science and Engineering: Taylor \& Francis)

[7] Katzke H, Bismayer U and Tolédano P 2006 Theory of the high-pressure structural phase transitions in Si, Ge, Sn, and Pb Physical Review B 73134105

[8] Gaál-Nagy K and Strauch D 2006 Transition pressures and enthalpy barriers for the cubic diamond $>\beta$-tin transition in Si and Ge under nonhydrostatic conditions Physical Review B 73134101

[9] Menoni C S, Hu J Z and Spain I L 1986 Germanium at high pressures Physical Review B 34 362-8

[10] Qadri S B, Skelton E F and Webb A W 1983 High pressure studies of Ge using synchrotron radiation Journal of Applied Physics 54 3609-11

[11] Mujica A and Needs R J 1993 First-principles calculations of the structural properties, stability, and band structure of complex tetrahedral phases of germanium: ST12 and BC8 Physical Review B 48 17010-7

[12] Wang J-T, Chen C, Mizuseki H and Kawazoe Y 2013 Kinetic Origin of Divergent Decompression Pathways in Silicon and Germanium Physical Review Letters 110 165503

[13] Coppari F, Chervin J C, Congeduti A, Lazzeri M, Polian A, Principi E and Di Cicco A 2009 Pressure-induced phase transitions in amorphous and metastable crystalline germanium by Raman scattering, $\mathrm{x}$-ray spectroscopy, and ab initio calculations Physical Review B $\mathbf{8 0} 115213$

[14] Malone B D and Cohen M L 2012 Electronic structure, equation of state, and lattice dynamics of low-pressure Ge polymorphs Physical Review B $\mathbf{8 6} 054101$

[15] Haberl B, Guthrie M, Malone B D, Smith J S, Sinogeikin S V, Cohen M L, Williams J S, Shen G and Bradby J E 2014 Controlled formation of metastable germanium polymorphs Physical Review B 89144111

[16] Nelmes R J, McMahon M I, Wright N G, Allan D R and Loveday J S 1993 Stability and crystal structure of BC8 germanium Physical Review B 48 9883-6

[17] Bates C H, Dachille F and Roy R 1965 High-Pressure Transitions of Germanium and a New High-Pressure Form of Germanium Science 147 860-2

[18] Brazhkin V V, Lyapin A G, Popova S V and Voloshin R N 1995 Nonequilibrium phase transitions and amorphization in $\mathrm{Si}, \mathrm{Si} / \mathrm{GaAs}, \mathrm{Ge}$, and $\mathrm{Ge} / \mathrm{GaSb}$ at the decompression of high-pressure phases Physical Review B $\mathbf{5 1}$ 7549-54 
[19] Renard C, Boukhicha R, Gardès C, Fossard F, Yam V, Vincent L, Bouchier D, Hajjar S, Bubendorff J L, Garreau G and Pirri C 2011 Size effect on Ge nanowires growth kinetics by the vapor-Liquid-Solid mechanism Thin Solid Films 520-8 3314-3318

[20] Voute A, Deutsch M, Kalinko A, Alabarse F, Brubach J B, Capitani F, Chapuis M, Ta Phuoc V, Sopracase R and Roy P 2016 New high-pressure/low-temperature set-up available at the AILES beamline Vibrational Spectroscopy 86 17-23

[21] Raffy C, Furthmüller J and Bechstedt F 2002 Properties of hexagonal polytypes of group-IV elements from first-principles calculations Physical Review B $\mathbf{6 6} 075201$

[22] Di Cicco A, Frasini A C, Minicucci M, Principi E, Itiè J-P and Munsch P 2003 Highpressure and high-temperature study of phase transitions in solid germanium physica status solidi (b) $\mathbf{2 4 0}$ 19-28

[23] Wang H, Liu J F, He Y, Wang Y, Chen W, Jiang J Z, Olsen J S and Gerward L 2007 High-pressure structural behaviour of nanocrystalline Ge Journal of Physics: Condensed Matter 19156217

[24] Olego D and Cardona M 1982 Pressure dependence of Raman phonons of Ge and 3CSiC Physical Review B 25 1151-60

[25] Welber B, Cardona M, Tsay Y-F and Bendow B 1977 Effect of hydrostatic pressure on the direct absorption edge of germanium Physical Review B 15 875-9

[26] Olijnyk H 1992 Raman scattering in metallic Si and Ge up to 50 GPa Physical Review Letters 68 2232-4

[27] Huston L Q, Johnson B C, Haberl B, Wong S, Williams J S and Bradby J E 2017 Thermal stability of simple tetragonal and hexagonal diamond germanium Journal of Applied Physics 122175108

[28] Zhao Z, Zhang H, Kim D Y, Hu W, Bullock E S and Strobel T A 2017 Properties of the exotic metastable ST12 germanium allotrope Nature Communications 813909

[29] Dash W C and Newman R 1955 Intrinsic Optical Absorption in Single-Crystal Germanium and Silicon at $77^{\circ} \mathrm{K}$ and $300^{\circ} \mathrm{K}$ Physical Review 99 1151-5

[30] Goi A R, Syassen K and Cardona M 1989 Direct-band-gap absorption in germanium under pressure Physical Review B 39 12921-4

[31] Ikoma Y, Toyota T, Ejiri Y, Saito K, Guo Q and Horita Z 2016 Allotropic phase transformation and photoluminescence of germanium nanograins processed by highpressure torsion Journal of Materials Science 51 138-43 
Figure 1

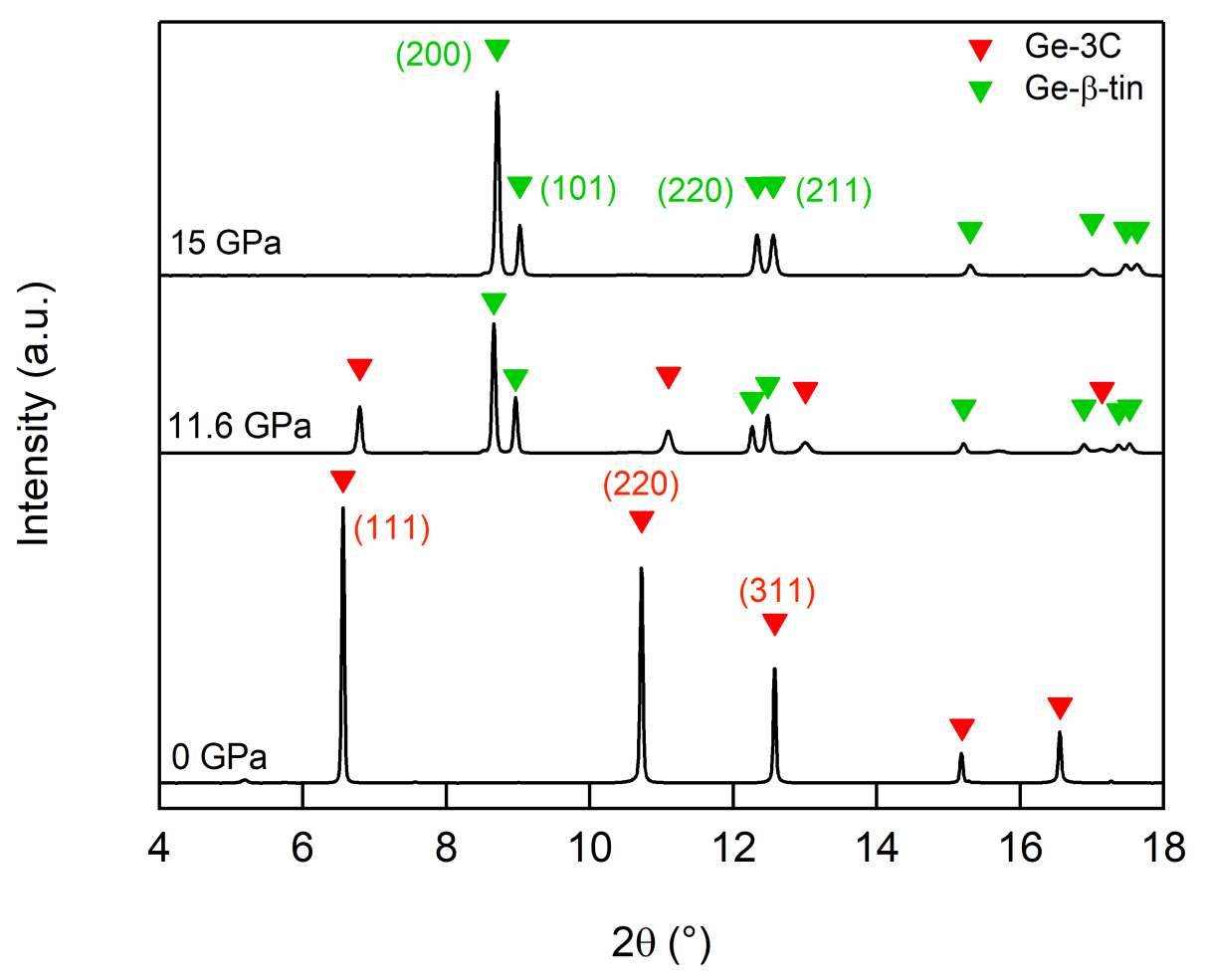


Figure 2

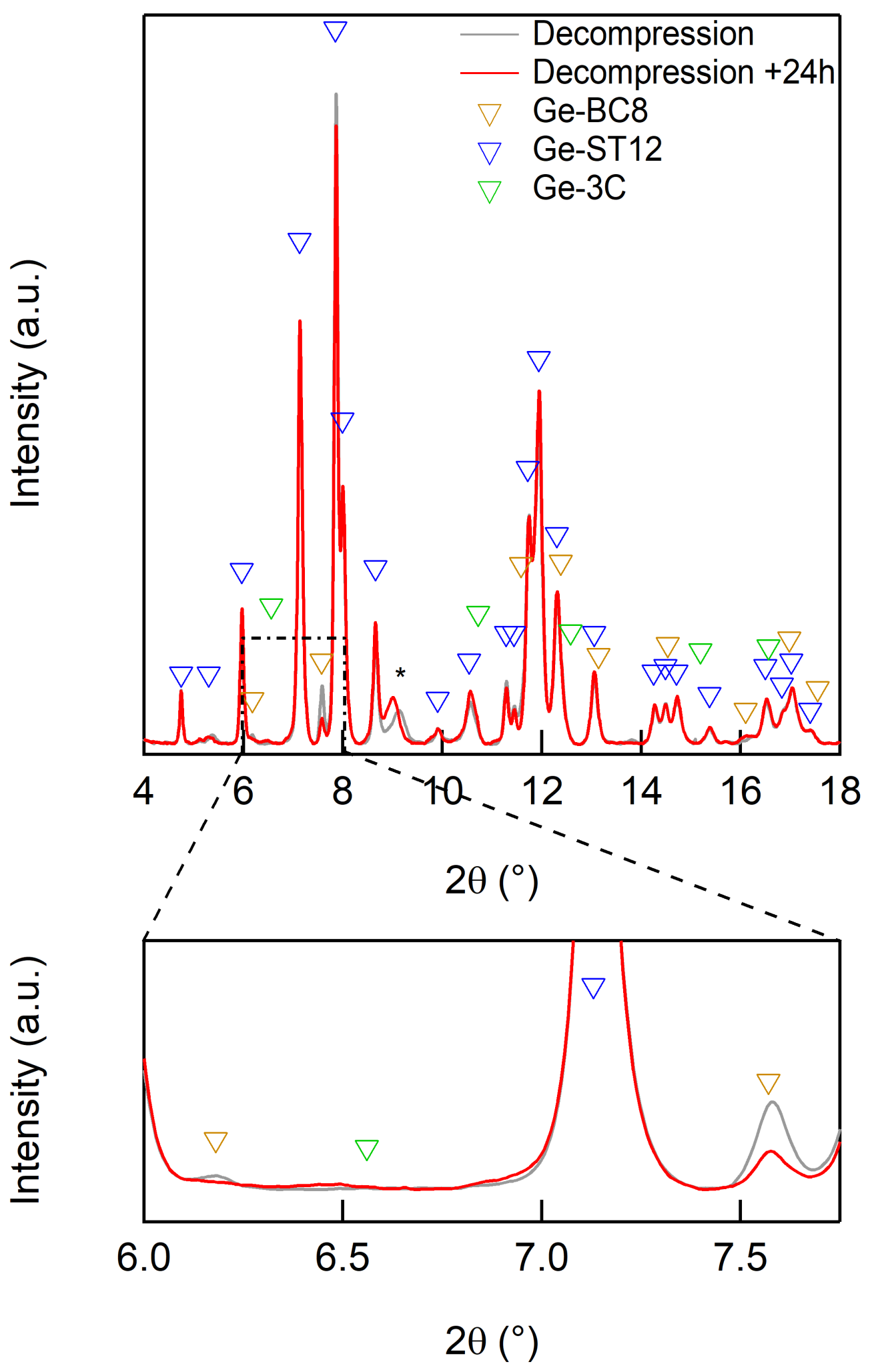


Figure 3

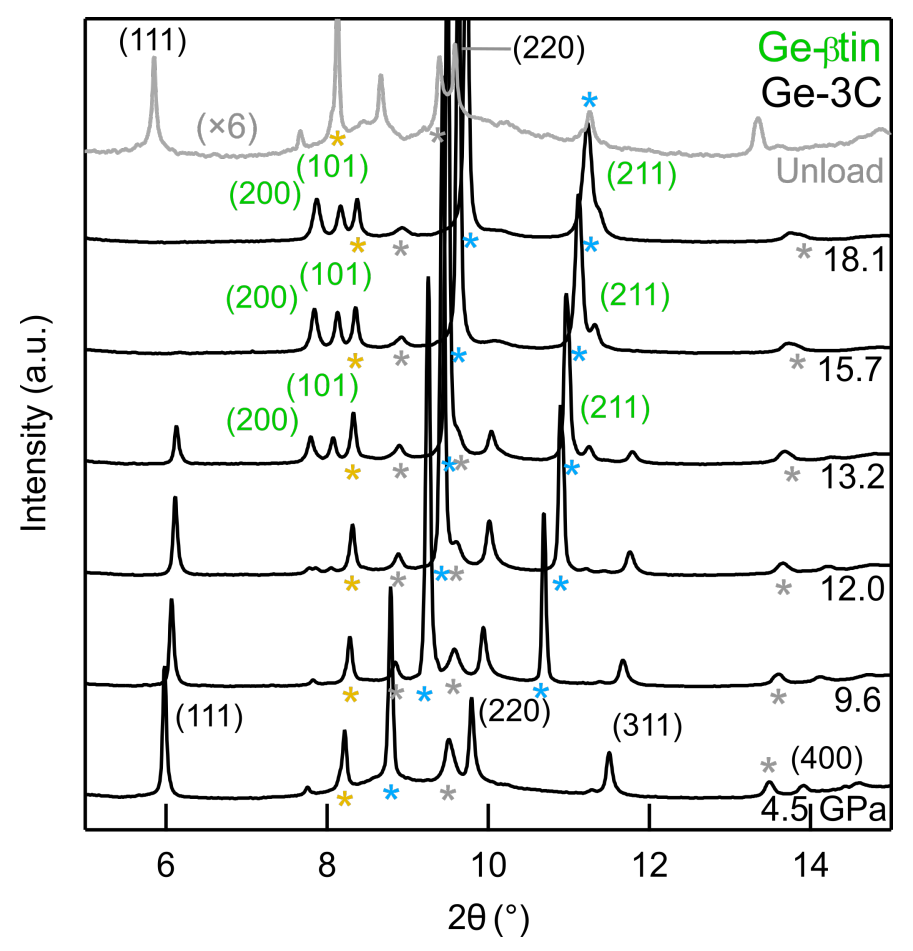


Figure 4
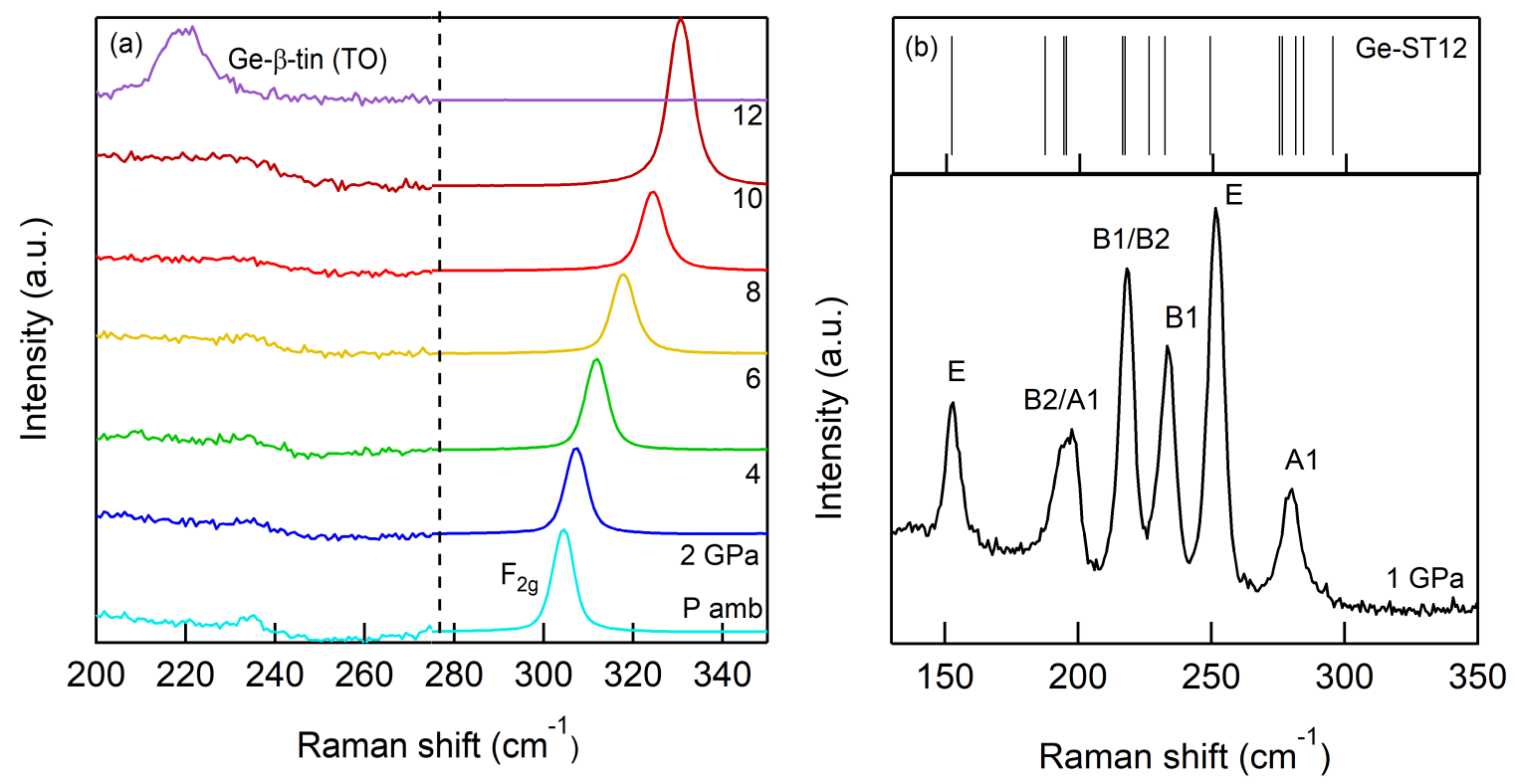
Figure 5
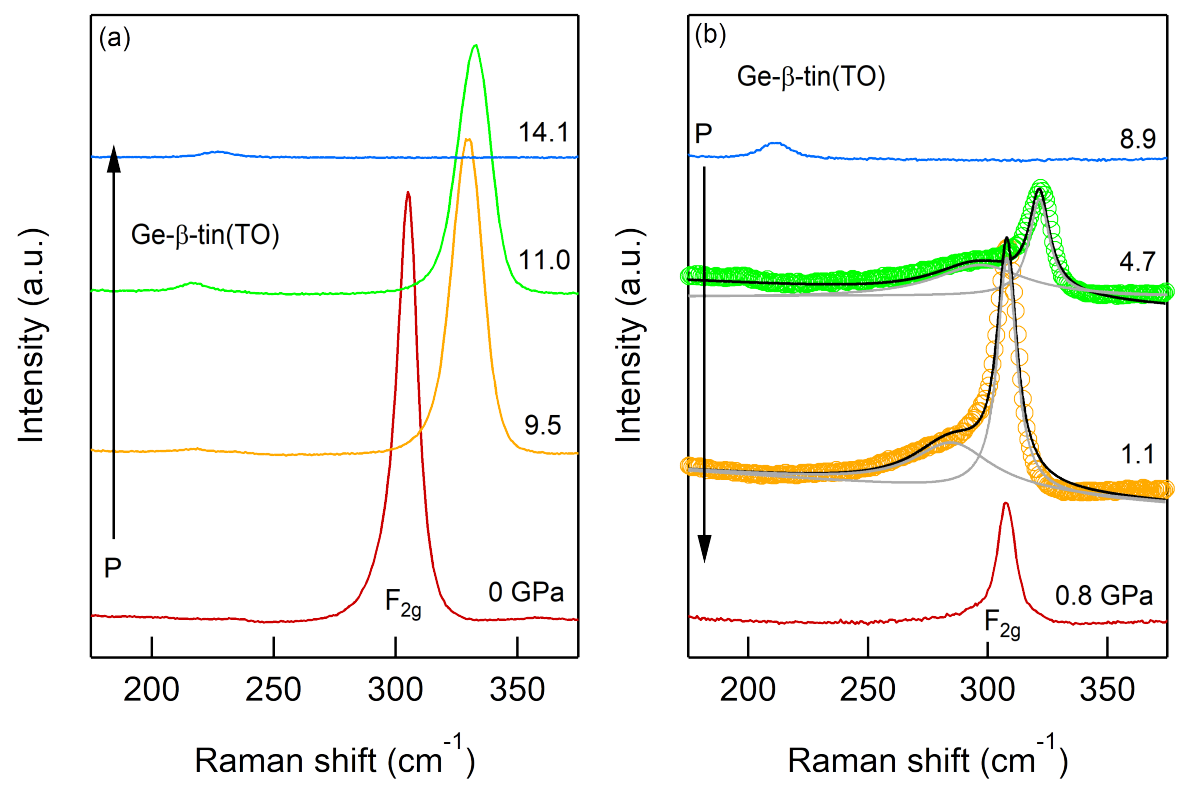
Figure 6
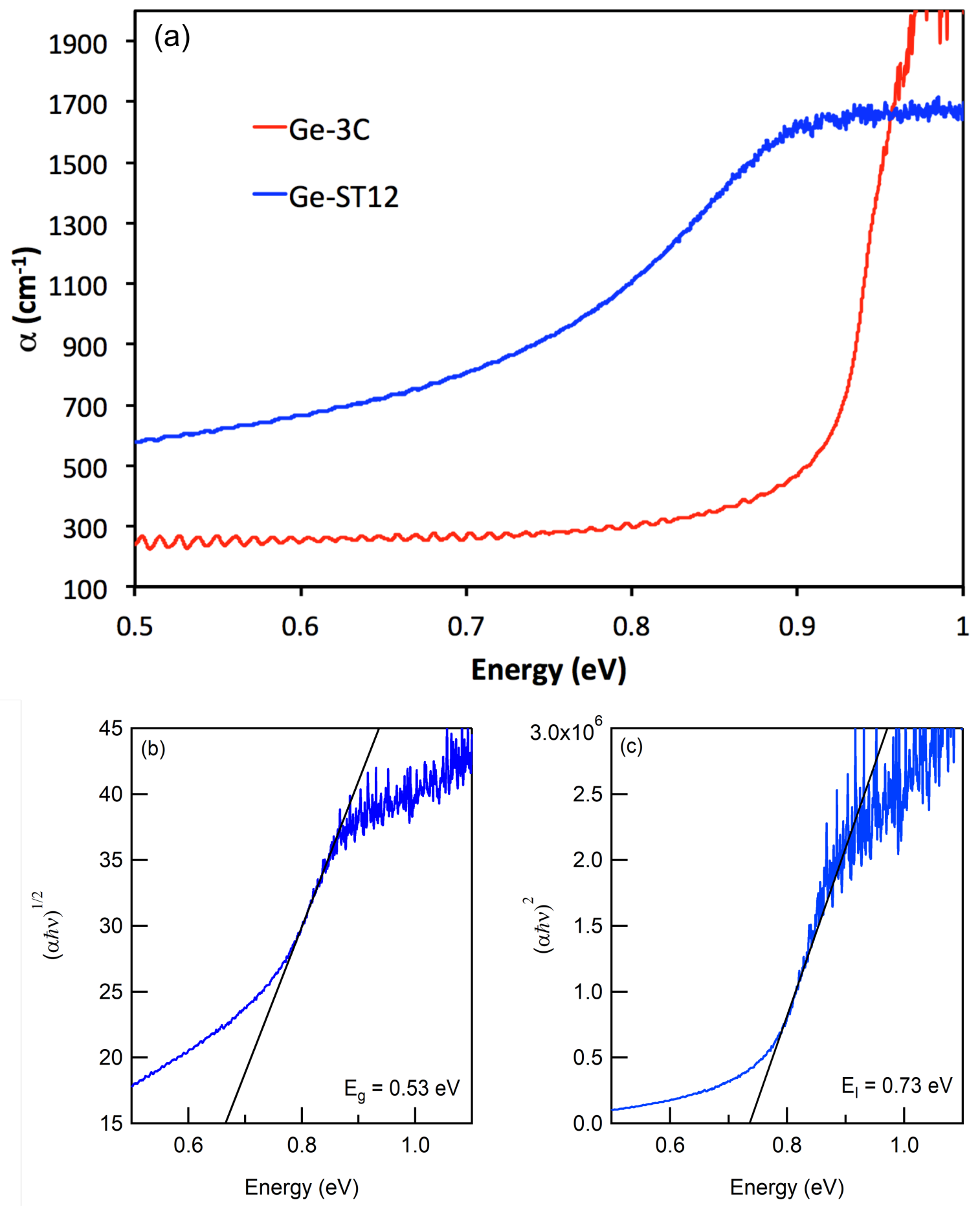
Figure 7

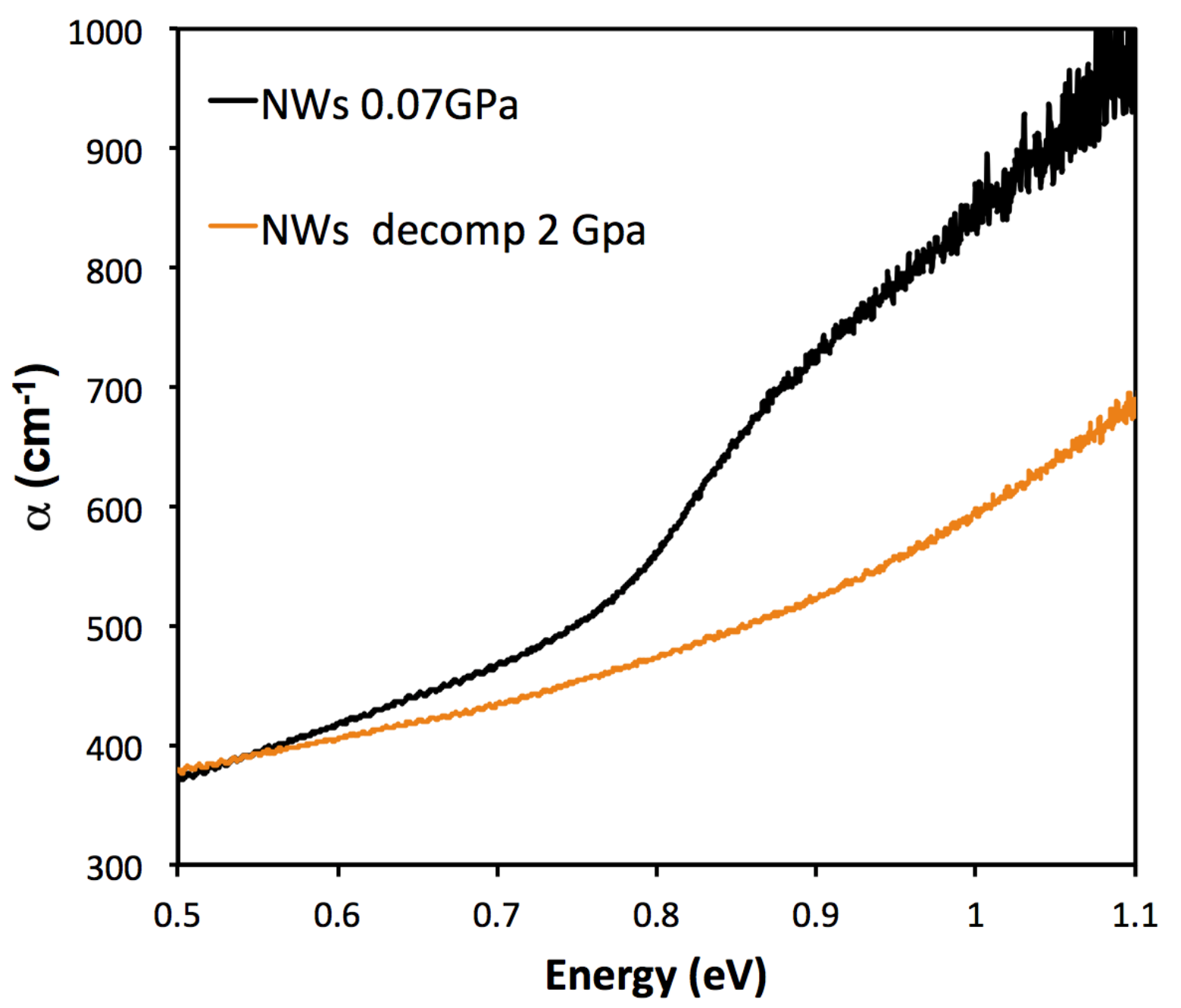

